\title{
Endoscopic Third Ventriculostomy and CyberKnife Radiosurgery of a Pineal Parenchymal Tumor of Intermediate Differentiation
}

Kevin Kwong-Hon Chow, Melanie H. Gephart, Jennifer Ziskin, Hannes Vogel, Gordon H. Li 1.

Corresponding author: Gordon H. Li, gordonli@stanford.edu
Received 10/30/2013 Review began 10/30/2013 Published 11/27/2013

\section{() Copyright 2013}

Chow et al. This is an open access article distributed under the terms of the Creative Commons Attribution License CC-BY 3.0., which permits unrestricted use, distribution, and reproduction in any medium, provided the original author and source are credited.
Categories: Radiation Oncology, Neurosurgery

Keywords: cyberknife, radiosurgery, pineal tumor

\section{Introduction}

Pineal parenchymal tumors (PPT) are rare neoplasms of the pineal gland which constitute less than $0.3 \%$ of all intracranial tumors [1]. They include, in order of World Health Organization (WHO) grade, pineocytomas (Grade I), pineal parenchymal tumors of intermediate differentiation (PPTID) (Grade II-III), and pineoblastomas (Grade IV) [2]. More recently described are papillary tumors of the pineal region (PTPR), which appear histologically distinct from PPTs and may represent a different cell of origin or an extension of the continuum of PPTs [3-4]. PPTs are usually diagnosed in younger patients, with a median age typically in the mid-30's [1,5]. A wide range of treatment strategies have been reported in the literature, which generally include some combination of surgery, radiation, or chemotherapy. Given the rarity of these tumors, optimal therapy has yet to be defined. Determining the optimal treatment strategy for an elderly patient with a PPT is particularly challenging, given the dearth of published studies on this cohort and the fact that these patients may not tolerate open surgery for resection of their tumor. Here, we describe an 82year-old patient with PPTID treated with endoscopic third ventriculostomy, endoscopic biopsy, and CyberKnife radiosurgery. Informed patient consent was obtained for all procedures.

\section{Case Presentation}

The patient is an 82-year-old with a past medical history significant for hypertension, hypothyroidism, and atrial fibrillation on Coumadin who presented after several months of progressive weakness, imbalance, memory deficits, and confusion. She reported that she had fallen multiple times in the past month, but denied any head trauma. She also denied headaches, nausea, vomiting, seizures, or focal neurological deficits. The patient had a normal neurological exam, with the exception of mild impairment in memory. Computerized tomography (CT) and magnetic resonance imaging (MRI) of the brain were obtained which showed a $2 \mathrm{~cm}$ pineal mass and hydrocephalus (Figure 1). The patient deteriorated while under observation in-house, becoming lethargic, waking up only to stimulation, and being oriented only to self.

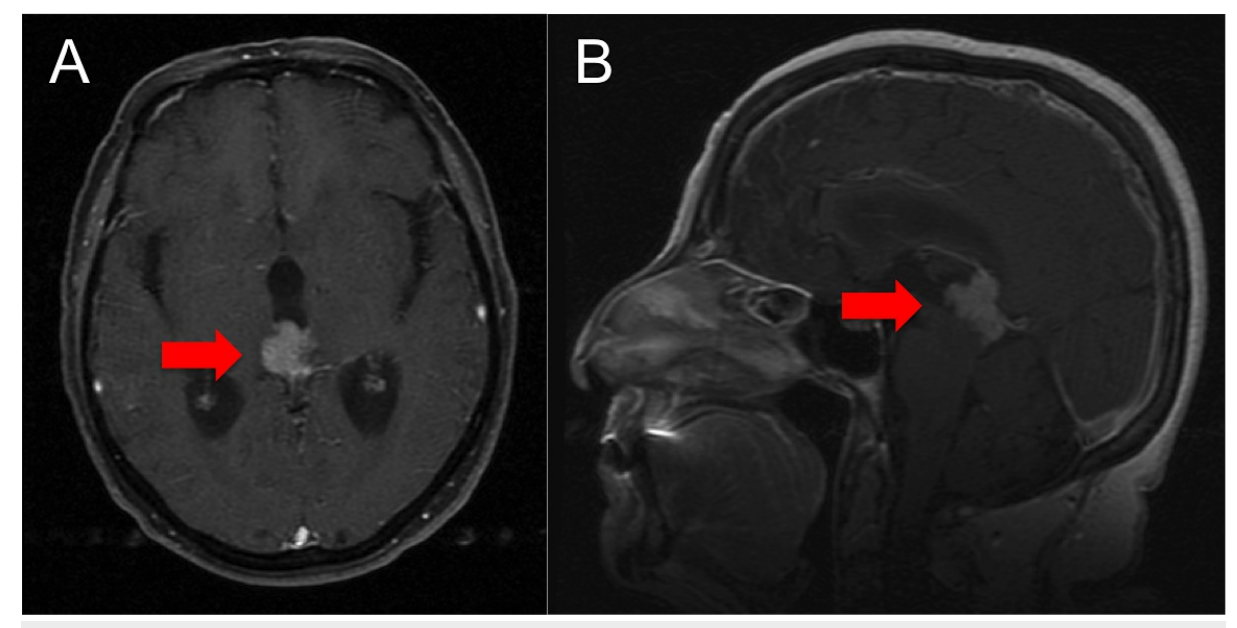




\section{Cureus}

FIGURE 1: Pre-operative MRI, T1 post-gadolinium, showing irregular shaped, contrast-enhancing lesion in the posterior third ventricle causing hydrocephalus, axial view (A) and sagittal view (B)

We proceeded with an endoscopic third ventriculostomy (ETV) to relieve her symptomatic hydrocephalus and an endoscopic tumor biopsy to inform subsequent treatment planning. The ETV was performed via Kocher's point on the right (Figure 2). After the ETV, endoscopic biopsy of the pineal mass was performed via a separate burr hole placed anterior to the right-sided Kocher's point burr hole. Direct visualization of a soft white tumor in the posterior third ventricle was achieved, and biopsy specimens were sent for permanent pathology. An external ventricular drain (EVD) was placed intra-operatively in the right frontal horn.

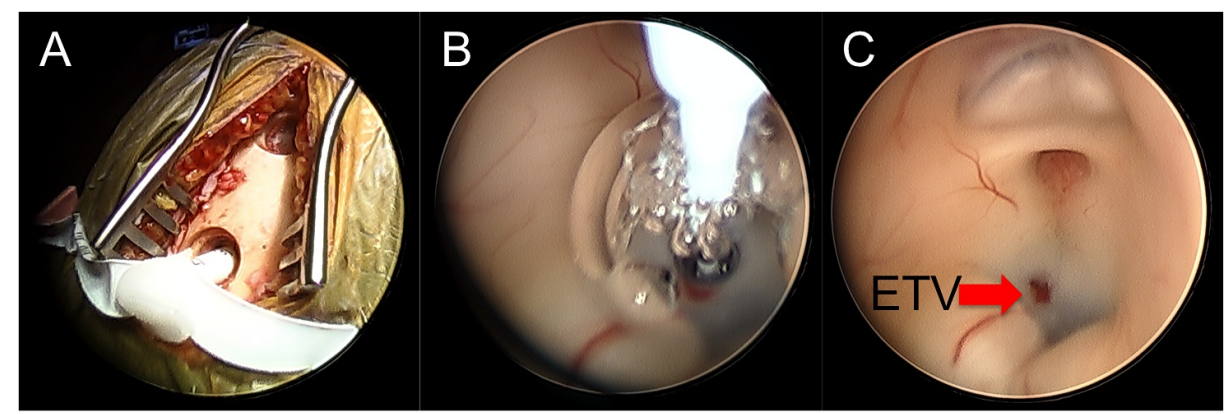

FIGURE 2: Intra-operative images of the burr hole placement, one at Kocher's point on the right, and one $3 \mathrm{~cm}$ anterior just behind the hairline (A).

A Bugbee wire was used to create a hole in the floor of the third ventricle after which an Integra balloon catheter was inserted and inflated to expand the venticulostomy (B). Completed third ventriculostomy (C)

Postoperatively, the patient had an uneventful recovery. A CT scan obtained after the surgery redemonstrated the pineal mass and showed slightly decreased ventricle size. The EVD was removed on postop day one. By the time of her first follow-up appointment one month from surgery, the patient showed significant improvement in her mental status and was noted to be fully oriented with improved strength, balance, and ability to ambulate independently. She reportedly had only mild difficulty with processing tasks (e.g. playing solitaire), but was otherwise markedly better than on admission. Histology revealed dense neoplastic cells with oval to angular nuclei and scant eosinophilic cytoplasm. Scattered apoptotic cells and focal areas of necrosis were identified. Three mitotic figures were noted per 10 high-power fields. The tumor was positive for synaptophysin and chromogranin, negative for CKMIX, and showed Ki-67 positivity of 5$10 \%$. These features are consistent with the diagnosis of pineal parenchymal tumor of intermediate differentiation (PPTID), WHO Grade II-III (Figure 3). 


\section{Cureus}

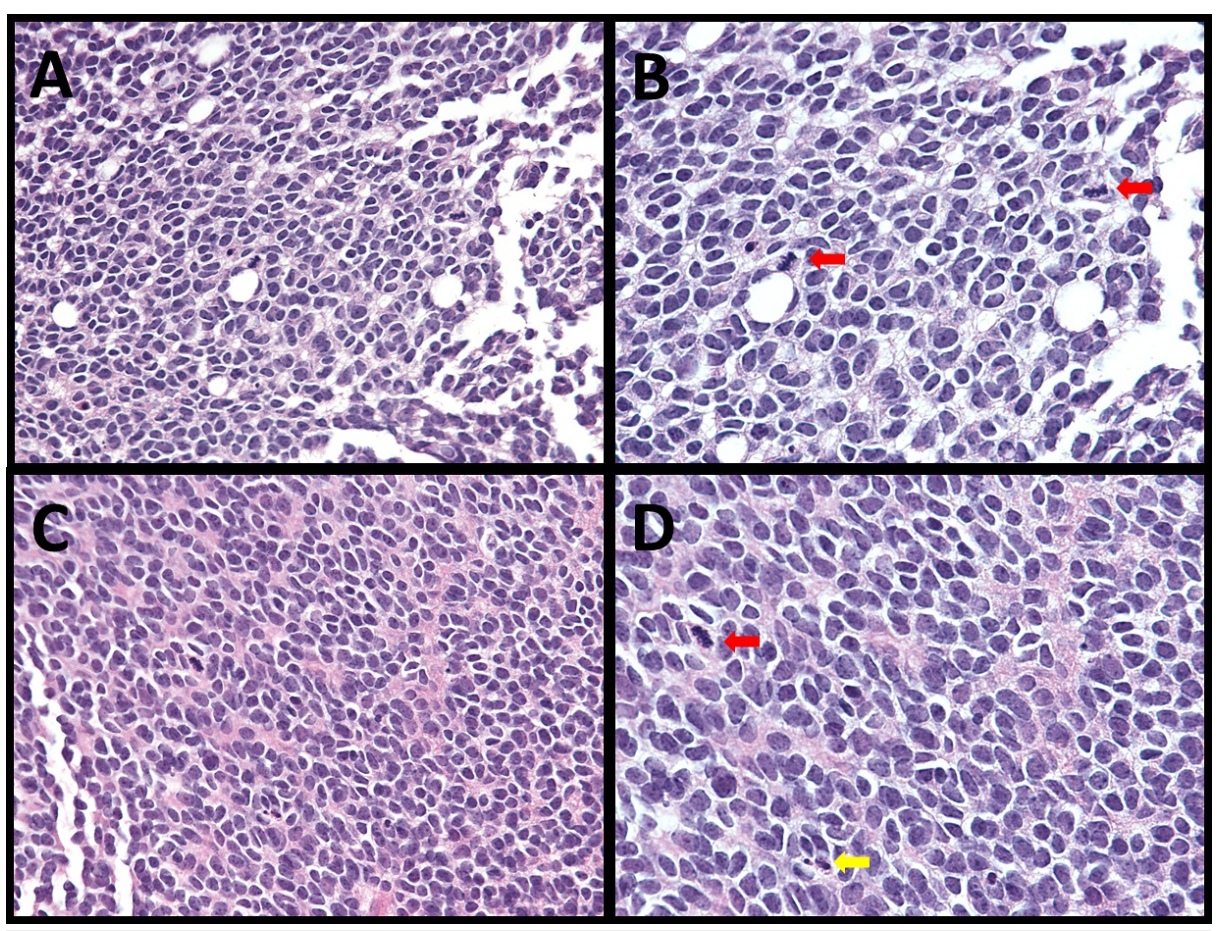

FIGURE 3: Histology of PPTID - hematoxylin and eosin stained slides at 40x ( $A$ and $C$ ) and 60x magnification ( $B$ and $D$ ) showing dense neoplastic cells with oval to angular nuclei and scant eosinophilic cytoplasm.

Red arrows indicate mitotic figures. Yellow arrow indicates an apoptotic cell.

Cytological examination of the CSF was negative for malignant cells and spine imaging revealed no drop metastases. After discussion of the patient's case at multidisciplinary tumor board, and with consideration of her age and comorbidities, the decision was made to defer craniospinal irradiation and surgical resection. Instead, the patient was offered treatment of the primary lesion with CyberKnife radiosurgery. The patient underwent further imaging for treatment planning and tumor contouring (Figure 4). The patient received a total dose of $24 \mathrm{~Gy}$ in three fractions. This radiosurgery dose balances the effects of tumor killing with the risk of causing radiation necrosis and injury to surrounding tissue. The patient tolerated the CyberKnife treatment with no adverse effects. 


\section{Cureus}

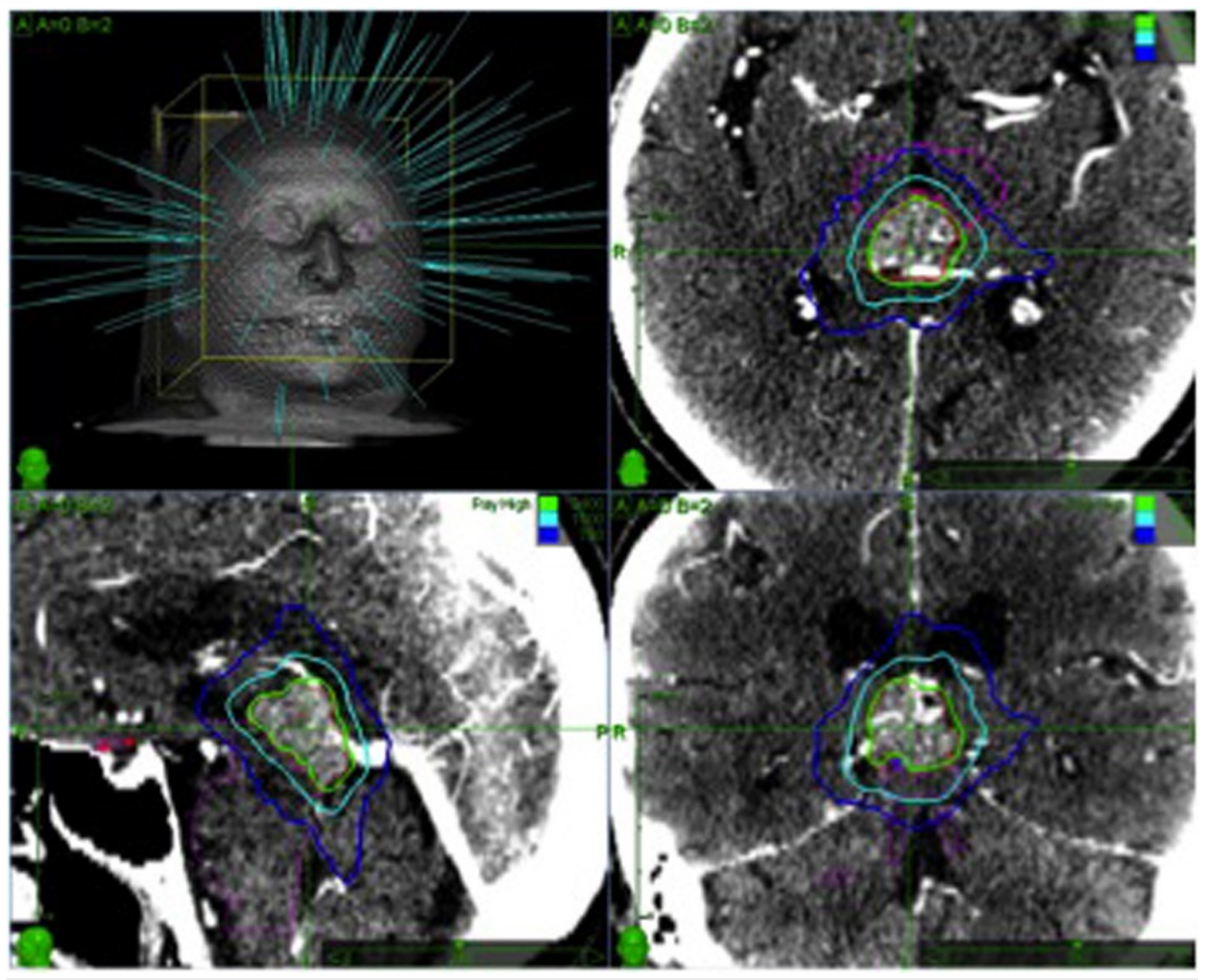

FIGURE 4: CyberKnife stereotactic radiosurgery treatment plan

\section{Discussion}

Tumors of the pineal region can take several forms. Most common are germ cell tumors; however, gliomas, ependymomas, choroid plexus papillomas, meningiomas, and the aforementioned PPTs also arise from this "seat of the soul" [6-8]. PPTs are extremely rare tumors, particularly in elderly patients. They generally carry a poorer prognosis than other tumors of the pineal region, and there is currently no consensus on what is the best standard of care [7].

Given the location of PPTs, obstructive hydrocephalus is a common finding with over $90 \%$ of patients presenting with obstruction in one series [9]. As such, many of these patients undergo placement of a ventriculoperitoneal (VP) shunt or ETV as their first intervention. Biopsy of the tumor and sampling of the CSF can be performed concomitantly with surgery to relieve the hydrocephalus as was done in this particular case. Having a tissue diagnosis is critical in helping to determine the management plan given the wide array of neoplasms that can occupy the pineal region.

Open or endoscopic-assisted surgical resection of PPTs remains an important treatment modality, given data that suggests that the amount of residual disease after initial treatment inversely correlates with overall survival [1]. Various approaches have been described to access the pineal region, and the appropriate approach should be chosen after careful consideration of the location of the tumor [10]. In the case of our 82-year-old patient with a history of hypertension and atrial fibrillation for which she takes Coumadin, it was felt that open surgery would be high risk, and therefore, other options were considered.

Chemotherapy has been studied as adjuvant therapy with multidrug regimens, including some combination of vincristine, lomustine, cisplatin, procarbazine, etoposide, cyclophosphamide, and others, producing partial responses in patients with PPTs [11]. However, the role of chemotherapy remains to be clearly defined given the rarity of these tumors and the lack of adequately powered randomized controlled trials.

Radiation therapy is appealing since it obviates the need for open surgical resection and has demonstrated potential for local tumor control. Fractionated external beam radiotherapy of pineocytomas and a PPTID with a median dose of $54 \mathrm{~Gy}$ directed to the primary tumor site has been shown to produce long-term remission (over seven years); patients with pineoblastoma, however, had poorer outcomes, developing local or spinal recurrence in spite of additional whole brain or craniospinal irradiation [12].

Stereotactic radiosurgery (SRS) involves high doses of radiation, historically in a single fraction, in an effort to destroy tumor cells and shrink the tumor. This is in contrast to conventional fractionated radiotherapy which exploits the differential sensitivity of tumor vs. normal cells to repeated exposure to lower doses 
of radiation. SRS has also been described for pineal region tumors and PPTs specifically. Gamma Knife radiosurgery, which involves the application of a skull mounted frame to achieve stereotaxis, has been shown to be particularly effective in controlling the growth of low-grade pineocytomas with reported rates of local tumor control of 85 to 100\% [9, 13]. Tumor control rates for the more aggressive PPTIDs and pineoblastomas was reported to be about $30 \%$ in one series [13]. CyberKnife stereotactic radiosurgery is a frameless system and has been used to successfully treat several intra- and extracranial tumors [14-15].

\section{Conclusions}

While there has been one reported case of CyberKnife treatment of a pineal germinoma in a child, the case presented here is, to our knowledge, the first use of CyberKnife to treat a pineal parenchymal tumor. This treatment modality was chosen as an alternative to surgical resection, given our patient's age and medical comorbidities. Follow-up will be needed to determine the long-term efficacy of this new treatment modality.

\section{Additional Information \\ Disclosures}

Human subjects: Consent was obtained by all participants in this study. Conflicts of interest: In compliance with the ICMJE uniform disclosure form, all authors declare the following: Payment/services info: All authors have declared that no financial support was received from any organization for the submitted work. Financial relationships: All authors have declared that they have no financial relationships at present or within the previous three years with any organizations that might have an interest in the submitted work. Other relationships: All authors have declared that there are no other relationships or activities that could appear to have influenced the submitted work.

\section{References}

1. Lutterbach J, Fauchon F, Schild SE, Chang SM, Pagenstecher A, Volk B, Ostertag C, Momm F, Jouvet A: Neurosurgery. 2002. Malignant pineal parenchymal tumors in adult patients: Patterns of care and prognostic factors, 51:44-55.

2. Louis DN, Ohgaki H, Wiestler OD, Cavenee WK, Burger PC, Jouvet A, Scheithauer BW, Kleihues P: The 2007 WHO classification of tumours of the central nervous system. Acta Neuropathol. 2007, 114:97-109.

3. Jouvet A, Fauchon F, Liberski P, Saint-Pierre G, Didier-Bazes M, Heitzmann A, Delisle MB, Biassette HA, Vincent S, Mikol J, Streichenberger N, Ahboucha S, Brisson C, Belin MF, Fevre-Montange M: Papillary tumor of the pineal region. Am J Surg Pathol. 2003, 27:505-512.

4. Cohan JN, Moliterno JA, Mok CL, Lavi E, Boockvar JA: Pineal parenchymal tumor of intermediate differentiation with papillary features: a continuum of primary pineal tumors? J Neurooncol. 2011, 101:301306.

5. Villa S, Miller RC, Krengli M, Abusaris H, Baumert BG, Servagi-Vernat S, Igdem S, Lucas A, Boluda S, Mirimanoff RO: Primary pineal tumors: Outcome and prognostic factors--a study from the Rare Cancer Network (RCN). Clin Transl Oncol. 2012, 14:827-834.

6. Baumgartner JE, Edwards MS: Pineal tumors. Neurosurg Clin N Am. 1992, 3:853-862.

7. Al-Hussaini M, Sultan I, Abuirmileh N, Jaradat I, Qaddoumi I: Pineal gland tumors: Experience from the SEER database. J Neurooncol. 2009, 94:351-358.

8. Santoro G, Wood MD, Merlo L, Anastasi GP, Tomasello F, Germano A: The anatomic location of the soul from the heart, through the brain, to the whole body, and beyond: A journey through Western history, science, and philosophy. Neurosurgery. 2009, 65:633-643.

9. Hasegawa T, Kondziolka D, Hadjipanayis CG, Flickinger JC, Lunsford LD: The role of radiosurgery for the treatment of pineal parenchymal tumors. Neurosurgery. 2002, 51:880-889.

10. Hart MG, Santarius T, Kirollos RW: How I do it--pineal surgery: Supracerebellar infratentorial versus occipital transtentorial. Acta Neurochir (Wien ). 2013, 155:463-467.

11. Schild SE, Scheithauer BW, Schomberg PJ, Hook CC, Kelly PJ, Frick L, Robinow JS, Buskirk SJ: Pineal parenchymal tumors. Clinical, pathologic, and therapeutic aspects. Cancer. 1993, 72:870-880.

12. Stoiber EM, Schaible B, Herfarth K, Schulz-Ertner D, Huber PE, Debus J, Oertel S: Long term outcome of adolescent and adult patients with pineal parenchymal tumors treated with fractionated radiotherapy between 1982 and 2003--A single institution's experience. Radiat Oncol. 2010, 5:122.

13. Mori Y, Kobayashi T, Hasegawa T, Yoshida K, Kida Y: Stereotactic radiosurgery for pineal and related tumors. Prog Neurol Surg. 2009, 23:106-118.

14. Calcerrada Diaz-Santos N, Blasco Amaro JA, Cardiel GA, Andradas AE: The safety and efficacy of robotic image-guided radiosurgery system treatment for intra- and extracranial lesions: A systematic review of the literature. Radiother Oncol. 2008, 89:245-253.

15. Peugniez C, Dewas S, Coche-Dequeant B, Leblond P, Defachelles AS, Thebaud E, Lacornerie T, Lartigau E: Use of conventional fractionation with CyberKnife in children: A report of 5 cases . J Pediatr Hematol Oncol. 2010, 32:472-475. 